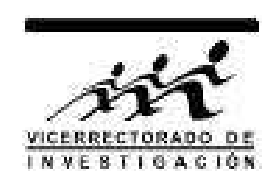

\title{
Transiciones intrabandas en superredes semiconductoras dimerizadas
}

\author{
D. I. Arrieta* y P. H. Rivera \\ Facultad de Ciencias Físicas, Universidad Nacional Mayor de San Marcos, Lima, Perú
}

Recibido 15 diciembre 2011 - Aceptado 30 marzo 2012

\begin{abstract}
En algunos dispositivos, el funcionamiento de los mismos está basado en las transiciones interbandas en la que los electrones y los huecos se recombinan emitiendo fotones en la región del visible e infrarrojo. En una superred se forman minibandas con determinados gaps entre los cuales se puede inducir transiciones intrabandas cuyos fotones emitidos se encuentren en la región del infrarrojo y las microondas. En el presente trabajo, desarrollamos un modelo que permite calcular las probabilidades por unidad de tiempo de tales transiciones y que nos permiten identificar las transiciones que se usan en los láseres de cascada cuántica.

Para ello, simulamos dos tipos de superredes, el primero con pozos de $100 \AA$ y el segundo con dos pozos dimerizados de 100 y $150 \AA$. En el primero, identificamos claramente tres transiciones para una transición de cascada y en el segundo, no se observa la posibilidad de transiciones en cascada. Pero, encontramos una posible evidencia de la competición del enmarañamiento cuántico de los autoestados pertenecientes a los pozos de 100 y $150 \AA$.
\end{abstract}

Palabras claves: Superredes semiconductoras, GaAs, AlGaAs, 2DEG, enmarañamiento cuantico.

\section{Intraband transitions in dimerized semiconductor superlattices}

In some devices, the functionning of them is based in the interband transitions in which the electrons and holes recombine emitting photons in the visible and infrared regions. In a superlattice is formed several minibands separated by minigaps, between them may be induced intraband transitions that emitting photons in the infrarred and microwaves regions. In the present work, we develop a model that permit us calculate the probabilities per unit time of such transitions and that permit us identify the transitions used in a quantum cascade laser.

We simulate two superlattice type, one with a $100 \AA$ quantum well and the second with two dimerized quantum wells of 100 and $150 \AA$. In the first case, we clearly identify three cascade transitions and, in the second, we do not observe the possibility of a quantum cascade transition. But, we find a possible evidence of the competition of entanglement states belonging to 100 and $150 \AA$ quantum wells.

Keywords: Semiconductor superlattices, GaAs, AlGaAs, 2DEG, entanglement states.

Desde aquellos tiempos en que fue sugerido las heteroestructuras semiconductoras [1] para el estudio del transporte electrónico en las superredes y en los diodos de tunelamiento resonante, las aplicaciones de estas se han desarrollado en una gran variedad de dispositivos que se encuentran en cualquier aparato que usamos en nuestra vida diaria desde los celulales, los pads, las calculadoras solares hasta los equipos médicos y militares de gran porte.

Por otro lado los láseres de estado sólido han explotado la emisión de fotones via la recombinación de pares electrones-huecos desde la banda de conducción hasta la banda de valencia 2. A diferencia de estos, los láseres de cascada cuántica son producidos por las transiciones intrabandas que ocurren en una superred construída de dos materiales semiconductores con bandgaps diferentes 3. Debido a la diferencia energética entre las bandas involucradas en las transiciones electrónicas, estos láseres emiten en la región del infrarrojo y fue observada experimentalmente por primera vez en 1994 [4].

El avance en las técnicas de crecimiento epitaxial ha permitido obtener heteroestructuras cuyos espesores son de muy pocas capas atómicas. Esto ha originado una discusión sobre la validez de la aproximación

*di-fis@hotmail.com 
de masa efectiva en la simulación de heteroestructuras con espesores de pocas capas atómicas [5]. En la que se reconsidera el papel de las matrices de transferencia para capas monoatómicas determinado por la conservación de la corriente y las propiedades de simetría de las monocapas.

En el presente trabajo, analizamos una superred formado por dos materiales semiconductores de diferentes bandgaps en la aproximación de la masa efectiva cuyo hamiltoniano es descrito semejante a un hamiltoniano tight-binding en la que el parámetro de hopping depende de la distancia entre dos puntos próximos del proceso de discretización. Incluímos perturbativamente el campo electromagnético que induce una interacción dipolar entre los niveles de energía de las minibandas de la superred.

\section{El modelo}

Una superred es una sucesión periódica de dos capas de materiales semiconductores de diferentes gaps de energía de modo que los electrones y huecos experimenten el confinamiento espacial de pozos (gap menor) y barreras ( gap mayor) en su acoplamiento con otros portadores de carga degenerados dentro de la misma superred. Esto significa que el potencial experimentado por los portadores de carga en su acoplamiento con otros portadores es uniforme, unidimensional y periódico, donde el periodo de la superred es la suma de los espesores de un pozo y una barrera.

Las superredes se clasifican en dos tipos, el primero se caracteriza porque el confinamiento espacial de los electrones y los huecos ocurren en la misma capa, mientras que, el segundo se caracteriza porque el confinamiento espacial de los electrones y huecos ocurren en capas diferentes 6, 6]

En la aproximación de la masa efectiva, el hamiltoniano del sistema se describe como $H=H_{x y}+H_{z}$, donde el hamiltoniano $H_{x y}$ corresponde al movimiento bidimensional de los portadores de carga y el hamiltoniano $H_{z}$ corresponde al movimiento unidimensional de los electrones a través de la heteroestructura cumpliendo la condición de autovalor con los autoestados acoplados de energía proyectados sobre los autoestados de posición en la dirección $x y$ y en la dirección $z$, respectivamente,

$$
\begin{gathered}
-\frac{\hbar^{2}}{2 m^{*}}\left(\frac{d^{2}}{d x^{2}}+\frac{d^{2}}{d y^{2}}\right)\langle x, y \mid E\rangle= \\
\frac{\hbar^{2}\left(k_{x}^{2}+k_{y}^{2}\right)}{2 m^{*}}\langle x, y \mid E\rangle, \\
{\left[-\frac{\hbar^{2}}{2 m^{*}} \frac{d^{2}}{d z^{2}}+V(z)\right]\left\langle z \mid E_{n}\right\rangle=E_{n}\left\langle z \mid E_{n}\right\rangle,}
\end{gathered}
$$

donde $E_{n}$ son los autovalores de energía en la dirección, $V(z)$ es la energía potencial de la heteroestructura, $m^{*}$ es la masa efectiva constante en el plano $x y$ y en la estructura correspondiente al pozo y a la barrera, $\hbar$ es la constante de Planck, $\langle x, y \mid E\rangle$ son las autofunciones de energía en el plano $x y$ y $\left\langle z \mid E_{n}\right\rangle$ son las autofunciones de energía en la dirección $z$. Para discretizar la Ec.(2) mediante el método de las diferencias finitas hacemos que $F(z)=\left\langle z \mid E_{n}\right\rangle$ de modo que los autovalores de posición quedan expresados como $z=a j$, donde $a$ es la distancia o métrica entre los dos puntos próximos o sucesivos y $j$ es un entero, luego un diferencial de la posición está dado por $d z=a d j$ que relaciona la variable contínua $z$ con la variable discreta $j$. Por tanto, la derivada es expresada como $d / d z=(1 / a)(d / d j)$ y aplicando a las autofunciones de energía se expresan como [8]

$$
\begin{aligned}
\frac{d F}{d z} & \rightarrow \frac{1}{a} \frac{d F}{d j}=\frac{1}{a}\left(F_{j+1}-F_{j}\right) \\
\frac{d^{2} F}{d z^{2}} & \rightarrow \frac{1}{a} \frac{d}{d j} \frac{1}{a} \frac{d F}{d j}=\frac{1}{a^{2}}\left(F_{j+1}-2 F_{j}+F_{j-1}\right) .
\end{aligned}
$$

En esta aproximación la ecuación de autovalor, Ec.(2), queda expresado como

$$
-t F_{j+1}+\left(2 t+V_{j}\right) F_{j}-t F_{j-1}=E_{j} F_{j}
$$

donde $t=\hbar^{2} / 2 m^{*} a^{2}$ y el hamiltoniano matricialmente está dado por

$$
H_{z}=\left(\begin{array}{ccccc}
\ddots & -t & 0 & 0 & 0 \\
-t & V_{-1}+2 t & -t & 0 & 0 \\
0 & -t & V_{0}+2 t & -t & 0 \\
0 & 0 & -t & V_{1}+2 t & -t \\
0 & 0 & 0 & -t & \ddots
\end{array}\right)
$$

el cual se asemeja al hamiltoniano tight binding de una cadena lineal de sítios atómicos donde los términos no diagonales iguales a $-t$ juega el rol del parámetro de hopping o la integral de sobrelapamiento de dos autofunciones de energía de dos sítios vecinos próximos, y los términos diagonales que expresan la energía de los orbitales atómicos de los sítios en la aproximación tightbinding, aquí en nuestro caso está dado por la energía potencial más $2 t$ en cada punto de discretización.

La dimensión de la matriz depende del número de puntos que deseamos discretizar toda la superred con las condiciones de contorno en que las autofunciones de energía deben converger a cero en los puntos extremos de la superred. La diagonalización depende del barrido de la energía entre el punto mínimo y el punto máximo de la banda de conducción del pozo y la barrera, respectivamente, para los electrones. Y entre el punto máximo y el punto mínimo de la banda de valencia de la barrera y del pozo, respectivamente, para los huecos. La diagonalización nos permite determinar los autovalores y las 
autofunciones de energía de los estados acoplados para todo el dispositivo. Se sabe que el número de autoestados por pozo, $n_{w}$, depende del ancho del pozo y de la altura de la barrera y si la superred tiene un número de pozos dados por $n_{p}$, entonces el número total de autoestados acoplados en el dispositivo es $N=n_{w} \times n_{p}$.

Cuando una radiación electromagnética incide sobre la heteroestructura induciendo transiciones entre los electrones que se encuentran en los autoestados acoplados que forman las minibandas de la superred en la banda de conducción, esta interacción entre la radiación y los electrones se describe mediante la aproximación de Peierls, donde el momento lineal sufre un corrimiento debido a la radiación de la forma $\boldsymbol{p} \rightarrow \boldsymbol{p}+e \boldsymbol{A}$, donde $\boldsymbol{A}$ es el potencial vectorial magnético, luego el hamiltoniano de interacción está dado por 9]

$$
H_{1}=\frac{e}{m^{*}} \boldsymbol{A} \cdot \boldsymbol{p}+\frac{e^{2}}{2 m^{*}} \boldsymbol{A}^{2}
$$

y el potencial vectorial magnético por 10 ]

$$
\begin{aligned}
\boldsymbol{A}(\boldsymbol{r})=\sum_{\boldsymbol{k}, \lambda} \sqrt{\frac{\hbar \mu_{0} c^{2}}{2 V \omega}}[ & a_{\boldsymbol{k}, \lambda} \boldsymbol{\varepsilon}(\boldsymbol{k}, \lambda) e^{i(\boldsymbol{k} \cdot \boldsymbol{r}-\omega t)}+ \\
& \left.+a_{\boldsymbol{k}, \lambda}^{\dagger} \boldsymbol{\varepsilon}(\boldsymbol{k}, \lambda) e^{-i(\boldsymbol{k} \cdot \boldsymbol{r}-\omega t)}\right],
\end{aligned}
$$

donde $a_{\boldsymbol{k}, \lambda}$ y $a_{\boldsymbol{k}, \lambda}^{\dagger}$ son los operadores de aniquilación y creación, respectivamete; $\varepsilon_{\boldsymbol{k}, \lambda}$ indica las dos polarizaciones de la radiación electromagnética; $\omega, \mu_{0}, V$ y $c$ son la frecuencia angular de la radiación electromagnética, la constante magnética, el volumen y la velocidad de la luz, respectivamente; mientras que el primer sumando de la Ec. (17) está asociado con la absorción de fotones por la superred, el segundo está con la emisión de los mismos.

El primer término del hamiltoniano de interacción, Ec.(6), basado en la forma del potencial vectorial magnético, Ec. (7), considera la absorción y emisión de un fotón, mientras que el segundo sumando considera la absorción y emisión de dos fotones.

Los electrones que se encuentran en los estados acoplados de la superred semiconductora se acoplan con la radiación electromagnética induciendo dipolos eléctricos que a su vez interacciona con el campo eléctrico de la propia radiación electromagnética que finalmente inducen las transiciones ópticas intrabandas así como las transiciones interbandas dependiendo de la energía de los fotones. Nuestro interés es estudiar las transiciones intrabandas de modo que la frecuencia de la radiación electromagnética sea del orden de la diferencia de dos autovalores de energía de las minibandas de conducción de la superred. La energía que involucra la transición intrabanda entre dos autoestados de energía es menor que la diferencia de energía entre la bandas de conducción del GaAs/AlGaAs, conocido como el band-offset,
$\Delta E_{c}$, de la banda de conducción, por tanto una solución perturbativa de la interacción considerando el primer sumando, absorbiendo o emitiendo un fotón, es considerada como una primera aproximación para el presente trabajo.

El método perturbativo dependiente del tiempo determina que las amplitudes de probabilidad de los autoestados de energía en la banda de conducción de una superred semiconductora, en la primera corrección, están dados por 9 ]

$$
c_{f}^{(1)}=-\frac{i}{\hbar} \int_{0}^{t} d t^{\prime} \exp \left[i \omega_{f i} t^{\prime}\right]\left\langle E_{f}^{(0)}\left|\hat{H}_{1}\left(t^{\prime}\right)\right| E_{i}^{(0)}\right\rangle
$$

donde $\omega_{f i}$ es la frecuencia de transición dado por

$$
\omega_{f i}=\frac{E_{f}^{(0)}-E_{i}^{(0)}}{\hbar},
$$

considerando que $E_{f}^{(0)}, E_{i}^{(0)}$ y $\left|E_{f}^{(0)}\right\rangle,\left|E_{i}^{(0)}\right\rangle$ son dos autovalores y dos autoestados acoplados de la superred, Ec. (5), que representan al estado inicial y final de la transición, respectivamente.

Como el potencial vectorial magnético en el hamiltoniano de interacción es una función periódica respecto al tiempo, a partir de la Ec.(8), obtenemos la regla de oro de Fermi, que mide la probabilidad por unidad de tiempo para un proceso de absorción o emisión, considerando la integral sobre todo el ángulo sólido y sumando todas las polarizaciones [9],

$$
R=\left.\sum_{\lambda} \int \frac{2 \pi}{\hbar}\left\langle E_{f}^{(0)}\left|H_{1}^{\prime}\right| E_{i}^{(0)}\right\rangle\right|^{2} \rho(\hbar \omega) d \Omega
$$

donde $H_{1}^{\prime} \sim H_{1}$ pues involucra al potencial vectorial magnético sin la parte temporal que ha sido integrada para obtener la Ec. (10) y $\rho$ es la densidad de estados de la radiación electromagnética.

Para el primer término del hamiltoniano $H_{1}^{\prime}$ tenemos que en $\boldsymbol{A} \cdot \boldsymbol{p}$, se debe especificar la polarización respecto al momento lineal de los portadores de carga. En la superred la dirección de cuantización está en la dirección $z$ y en las direcciones $x y$ están los electrones bidimensionales correspondiendo a cada pozo. Si consideramos que el vector $\boldsymbol{k}$ de la radiación electromagnética coincide con la dirección $z$ de cuantización de los portadores de carga entonces las direcciones de las polarizaciones $\varepsilon(\boldsymbol{k}, \lambda=1)$ y $\varepsilon(\boldsymbol{k}, \lambda=2)$ coinciden con las direcciones $x$ e $y$, respectivamente, de los gases bidimensionales. Por tanto, el producto $\boldsymbol{A} \cdot \boldsymbol{p}=A_{1} p_{x}+A_{2} p_{y}$, en la dirección $z$ no tiene ninguna contribución.

Para observar una contribución de la cuantización en el eje $z$ en las transiciones inducidas por el campo electromagnético debemos incidir la radiación paralela a la dirección $y, \boldsymbol{k}=\left(0, k_{y}, 0\right)$, luego las componentes 
del potencial vectorial $A_{1}\left(k_{y}, 1\right)$ es paralela a la dirección $z$ y $A_{2}\left(k_{y}, 2\right)$ es paralela a la dirección $x$, luego

$$
\boldsymbol{A} \cdot \boldsymbol{p}=A_{1}\left(k_{y}, z\right)(-i \hbar) \frac{d}{d z}+A_{2}\left(k_{y}, x\right)(-i \hbar) \frac{d}{d x} \text {. }
$$

Por tanto, los términos que multiplica a la absorción y emisión de fotones son los asociados a la onda plana en la dirección $y$, tales como $e^{i k_{y} y}, e^{-i k_{y} y}, e^{i 2 k_{y} y}$ $\mathrm{y} e^{-i 2 k_{y} y}$. Como las dimensiones en $x y$ pueden ser del orden de $z \sim 4000 \AA$, las energías involucradas en las transiciones en la dirección $z$ son del orden de $50 \mathrm{meV}$, como $E=\hbar c k$, donde la constante $\hbar c=1973269.631$ meV-A, luego $\lambda=39465.39$ Ay $k=2.534 \times 10^{-5} \AA^{-1}$ y $k z \sim k y \sim 0.1014<1$, con la inclusión de un mínimo error podemos considerar para la emisión y absorción que $e^{ \pm i k_{y} y} \approx 1$.

Considerando la densidad de estados para la radiación electromagnética, el número de estados fotónicos por unidad de energía [9, como

$$
\rho=\frac{V}{(2 \pi)^{3}} \frac{\omega^{2}}{\hbar c^{3}},
$$

finalmente la absorción y emisión están dados por

$$
\begin{aligned}
R=\frac{1}{2 \pi} & \left(\frac{e^{2} \hbar \mu_{0}}{m^{* 2} c}\right) \omega m_{f} \times \\
& \left|\left\langle m_{f}^{\prime}\left|\otimes\left\langle E_{f}^{(0)}\left|\frac{d}{d \hat{z}}\right| E_{i}^{(0)}\right\rangle \otimes\right| m_{f}-1\right\rangle\right|^{2}
\end{aligned}
$$

y

$$
\begin{aligned}
& R=\frac{1}{2 \pi}\left(\frac{e^{2} \hbar \mu_{0}}{m^{* 2} c}\right) \omega\left(m_{f}+1\right) \times \\
& \quad\left|\left\langle m_{f}^{\prime}\left|\otimes\left\langle E_{f}^{(0)}\left|\frac{d}{d \hat{z}}\right| E_{i}^{(0)}\right\rangle \otimes\right| m_{f}+1\right\rangle\right|^{2},
\end{aligned}
$$

respectivamente, donde la frecuencia de la radiación electromagnética $\omega=\omega_{f i}$, la frecuencia de transición, Ec.(9), entre los diferentes autoestados de las minibandas de la superred y $m_{f}$ es el número de fotones.

La relación que se establece entre las Ecs. 113, 14) y las soluciones de la Ec. (15) es a través de la condición de completez de los autoestados de posición en la dirección $z$, de la forma

$$
\begin{gathered}
\left\langle E_{f}^{(0)}\left|\frac{d}{d z}\right| E_{i}^{(0)}\right\rangle=\int d z^{\prime} \int d z\left\langle E_{f}^{(0)} \mid z^{\prime}\right\rangle\left\langle z^{\prime}\left|\frac{d}{d \hat{z}}\right| z\right\rangle\left\langle z \mid E_{i}^{(0)}\right\rangle \\
=\int d z^{\prime} \int d z\left\langle E_{f}^{(0)} \mid z^{\prime}\right\rangle \frac{d}{d z^{\prime}} \delta\left(z-z^{\prime}\right)\left\langle z \mid E_{i}^{(0)}\right\rangle \\
=\int d z\left\langle E_{f}^{(0)} \mid z\right\rangle \frac{d}{d z}\left\langle z \mid E_{i}^{(0)}\right\rangle \rightarrow \\
\text { discretizando } \rightarrow \sum_{j} \frac{1}{a} G_{j}^{*}\left(F_{j+1}-F_{j}\right)
\end{gathered}
$$

donde $F=\left\langle z \mid E_{i}^{(0)}\right\rangle$ y $G=\left\langle z \mid E_{f}^{(0)}\right\rangle$ son los autovectores del hamiltoniano $H_{z}$, Ec.(5), proyectados en el espacio de los autoestados de posición en la dirección $z$.

\section{Resultados y discusión}

\section{superredes y superredes dimerizadas}

Consideramos para el presente trabajo, en primer lugar, una superred conformada por veinte pozos cuánticos de GaAs con $150 \AA$ de espesor cada uno y por diecinueve barreras intermediarias de $\mathrm{Al}_{0.35} \mathrm{Ga}_{0.75}$ As de 10 $\AA$ de ancho cada uno, ambos rodeados de dos barreras laterales de $\mathrm{Al}_{0.35} \mathrm{Ga}_{0.75}$ As de $310 \AA$ que permiten que las autofunciones de onda en los extremos convergan y sean iguales a cero. La masa efectiva de los electrones en el GaAs es $m_{w}^{*}=0.067 m_{0}$, donde $m_{0}$ es la masa del electrón libre, mientras que para una concentración de $35 \%$ de Al la masa efectiva de los electrones en el $\mathrm{Al}_{0.35} \mathrm{Ga}_{0.75} \mathrm{As}$ es $m_{b}^{*}=0.096 m_{0}$. Para la misma concentración de Al la altura del potencial de las barreras o el band-offset de la banda de conducción es $\Delta E_{c}=301$ meV. Los parámetros dados aquí están en el centro de simetría $\Gamma$ donde el GaAs y $\mathrm{Al}_{0.35} \mathrm{Ga}_{0.75} \mathrm{As}$ poseen un band gap directo entre las bandas de conducción y valencia.



Figura 1: Banda de conducción de una superred de GaAs/ $\mathrm{Al}_{0.35} \mathrm{Ga}_{0.75}$ As con barreras de $10 \AA$, y pozos cuánticos de $150 \AA$ A . Se muestran los autoestados extremos inferior y superior de tres minibandas y el extremo inferior de la cuarta minibanda que en la presente configuración es incompleta porque solo tiene 12 autoestados ligados.

En la figura 1 se muestra la estructura electrónica de la banda de conducción de la superred mostrando los autoestados extremos, inferiores y superiores, de las tres minibandas claramente identificadas y el extremo inferior de una cuarta minibanda con 12 autoestados ligados. Cabe mencionar que las minibandas completas están conformados por 20 autoestados que corresponde a los estados acoplados de los 20 pozos cuánticos. El primer mini-gap entre el fondo de la banda de conducción y la primera minibanda formada es $E_{\mathrm{mg}_{1}}=15.22 \mathrm{meV}$, el 
segundo mini-gap entre la primera y segunda minibanda es $E_{\mathrm{mg}_{2}}=37.47 \mathrm{meV}$, mientras que el tercero entre la tercera y cuarta minibanda es $E_{\mathrm{mg}_{3}}=43.31 \mathrm{meV}$ y finalmente, el cuarto mini-gap entre la cuarta minibanda y la quinta minibanda incompleta es $E_{\mathrm{mg}_{4}}=34.46$ meV.

Por otro lado, también se observa que el ancho de las tres minibandas completas son $\Delta_{1}=7.61 \mathrm{meV}$, $\Delta_{2}=30.90 \mathrm{meV}$ y $\Delta_{3}=70.09 \mathrm{meV}$, respectivamente.

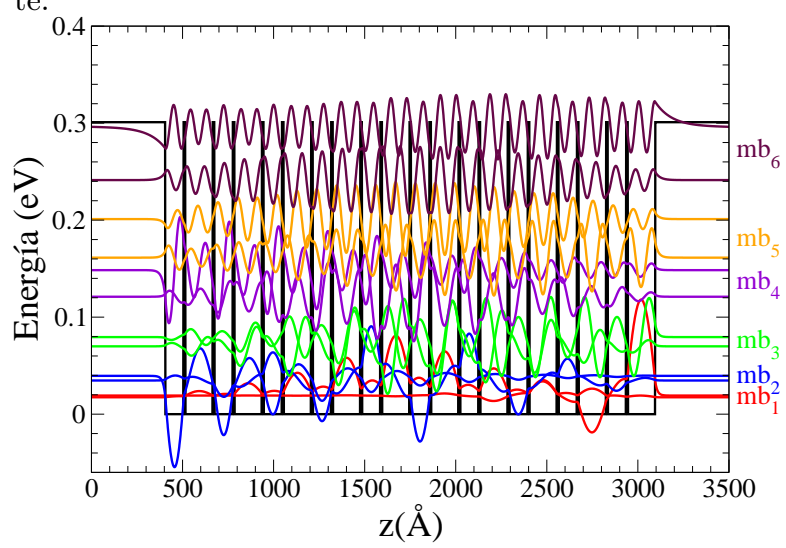

Figura 2: Banda de conducción de una superred dimerizada de $\mathrm{GaAs} / \mathrm{Al}_{0.35} \mathrm{Ga}_{0.75}$ As con barreras de $10 \AA$, y pozos cuánticos de 100 y $150 \AA$, que se repiten secuencialmente. Se muestran los autoestados extremos inferiores y superiores de seis minibandas con diez autoestados cada una.

La segunda clase de superred que estudiamos es la superred dimerizada que consta de dos pozos cuánticos de $100 \AA$ y $150 \AA$ separados por una barrera de $10 \AA$ que se repiten sucesivamente y a la cual designamos por ABAB...ABAB, este tipo de superred se muestra en la figura 2. Se observan seis minibandas cada una con diez autoestados acoplados separados desde el fondo de la banda de conducción con los siguientes mini-gaps, $E_{\mathrm{mg}_{1}}=17.38 \mathrm{meV}, E_{\mathrm{mg}_{2}}=15.52 \mathrm{meV}$, $E_{\mathrm{mg}_{3}}=30.43 \mathrm{meV}, E_{\mathrm{mg}_{4}}=41.59 \mathrm{meV}, E_{\mathrm{mg}_{5}}=12.89$ $\mathrm{meV} \mathrm{y} E_{\mathrm{mg}_{6}}=49.19 \mathrm{meV}$. Entre el segundo y el cuarto mini-gap existe un crecimiento en energía pero el quinto mini-gap decrece abruptamente para luego continuar el crecimiento con el sexto mini-gap. Esto tiene que ver con las autoenergías de los pozos cuánticos de 100 A y $150 \AA$, que se aproximan para esos valores de energía. Veamos, cuantitativamente se observa que el pozo de $100 \AA$ posee tres autovalores de energía dados por 41.48 $\mathrm{meV}, 155.45 \mathrm{meV}$ y $285.15 \mathrm{meV}$, el pozo de $150 \AA$ posee cuatro autovalores de energía de $20.41 \mathrm{meV}, 80.12 \mathrm{meV}$, $172.65 \mathrm{meV}$ y $274.80 \mathrm{meV}$ y el sistema dimerizado posee seis autovalores de energía de $19.28 \mathrm{meV}, 38.81 \mathrm{meV}$, $77.77 \mathrm{meV}, 139.70 \mathrm{meV}, 181.95 \mathrm{meV}$ y $263.64 \mathrm{meV}$. Se aprecia que el cuarto (139.70 meV) y el quinto (181.95 $\mathrm{meV}$ ) autovalor de energía del sistema dimerizado co- rresponde al segundo autovalor $(155.45 \mathrm{meV})$ del pozo de $100 \AA$ y al tercero $(172.65 \mathrm{meV})$ del pozo de $150 \AA$.

Los anchos de las minibandas de las superredes dimerizadas se muestran en la tabla 1, así como los minigaps formados entre dichas minibandas. Se puede observar en la figura 2, que los autoestados tienen una asimetría desplazada hacia la derecha esto se debe a la dimerización $\mathrm{ABAB}$... ABAB; hemos observado también, que las superredes BABA... BABA poseen una asimetría desplazada hacia la izquierda. Haciendo una dimerización incompleta $\mathrm{ABAB}$... ABA y BABA... BAB los autoestados son simétricos en todas las minibandas, recordemos que $\mathrm{A}$ corresponde a un pozo de $100 \AA$ y B corresponde a un pozo de $150 \AA$, ambos separados por una barrera de $10 \AA$.

\section{Absorción y emisión de las superredes}

Las probabilidades de absorción y emisión por unidad de tiempo son calculados usando las ecuaciones 13. 14] y 15) para una estructura de banda de la superred mostrada en la figura 1. Las transiciones entre la primera y segunda minibanda, Fig.3(a), así como entre la segunda y tercera minibanda, Fig.3(e), muestran veinte eventos con mayor probabilidad de las 400 posibles transiciones de absorción. Se observa que la distribución de probabilidades de las figuras 3(a) y 3(e) son completamente diferentes a la distribución de probabilidades entre gaussiana y lorenztiana mostrada por la figura 3(b) que corresponde a las transiciones entre la primera y la tercera minibanda. En la figura 3(f) se muestran las tres distribuciones simultáneamente para las transiciones de absroción y claramente se observa que la distribución de probabilidades con mayor amplitud y mayor espectro de energía es la que corresponde a las transiciones entre la segunda y la tercera minibanda. Esto se explica por el hecho de que el ancho de estas minibandas son mayores respecto a la primera minibanda, osea que ambas minibandas tienen una mayor dispersión que la primera minibanda. En la primera minibanda los autoestados de energía están muy localizados pues el ancho de la minibanda es de apenas $7.61 \mathrm{meV}$, ver tabla 1 .

La distribución de las probabilidades por unidad de tiempo para la absorción entre la segunda y la tercera minibanda que se muestra en la Fig.3(f) se ajusta aproximadamente a una serie polinómica dado por $R=$ $9.0108-0.095721 \omega+4.2685 \times 10^{-4} \omega^{2}-6.0515 \times 10^{-7} \omega^{3}$, mientras que a la distribución de probabilidades por unidad de tiempo para la absorción entre la primera y la segunda minibanda que se muestra en la Fig.3(a) se ajusta $R=3.475-0.072878 \omega+6.006 \times 10^{-4} \omega^{2}-$ $1.5955 \times 10^{-6} \omega^{3}$.

La vida media de los estados excitados en la superred varían desde 0.11 hasta 0.23 us para la tercera 
minibanda y desde 0.51 hasta 0.83 us para la segunda minibanda. Siendo las probabilidades por unidad de tiempo mayores para alcanzar la tercera minibanda, las transiciones entre esta minibanda y la segunda también son mayores de modo que pueden poblarse con más electrones hasta crear una emisión estimulada entre la segunda y la primera minibanda. En la Fig.3(c),(h) se muestran las probabilidades de emisión por unidad de tiempo entre la segunda y la primera minibanda, y entre la tercera y la segunda minibanda. Las formas de las distribuciones son las mismas que las de la absorción, pero en magnitud son el doble porque estamos considerando la emisión estimulada. La emisión espontánea es de la misma magnitud que el de la absorción.
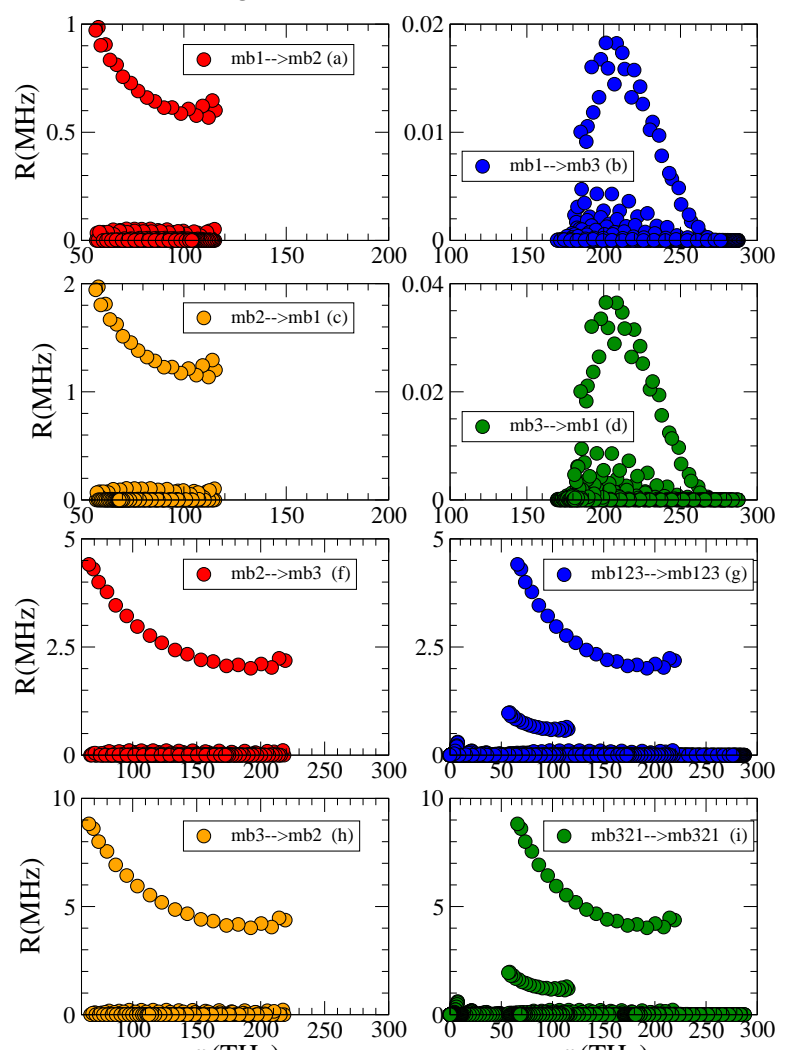

$\omega(\mathrm{THz})$

Figura 3: Las probabilidades de absorción (a), (b), (f), (g) y de emisión (c), (d), (h), (i) por unidad de tiempo son mostradas. Las transiciones de absorción entre las minibandas 1 y 2 (a), 2 y 3 (f) presenta la misma distribución de probabilidad a diferencia de la transición entre las minibandas 1 y 3 que presenta una distribución gaussiana. Lo mismo se muestra para las transiciones de emisión. Todas las transiciones posibles están mostradas en (g) para la absorción y en (i) para la emisión estimulada.

Aplicando una diferencia de potencial, tanto positiva como negativa, entre las dos barreras extremas de

las superredes observamos que para $\pm 5 \mathrm{mV}$ la distribución de probabilidades por unidad de tiempo mantiene la serie polinómica acompañado de distribuciones de probabilidades de mediana amplitud en el rango entre 50 y $220 \mathrm{THz}$, Fig.4(a,b). Se observa que para ambos voltajes, positivo y negativo, se mantiene una simetría en las distribuciones de las probabilidades temporales. Aumentando la diferencia de potencial para $\pm 10 \mathrm{mV}$ y $\pm 50 \mathrm{mV}$, Fig.4(b,c,d,e,f), se observa tres distribuciones con picos bien definidos a 10, 70 y $210 \mathrm{THz}$, esto significa que en este rango de voltajes tenemos una buena emisión estimulada para los láseres de cascada cuántica a $6.582,46.074$ y $138.222 \mathrm{meV}$. Para voltaje mayores a $50 \mathrm{mV}$ las minibandas comienzan a superponerse de modo que la superred para $100 \mathrm{mV}$ está prácticamente metalizada, aunque las transiciones a 10 y $210 \mathrm{THz}$ se mantienen pero la transición a $70 \mathrm{THz}$ se intensifica más, Fig.4(g,h).
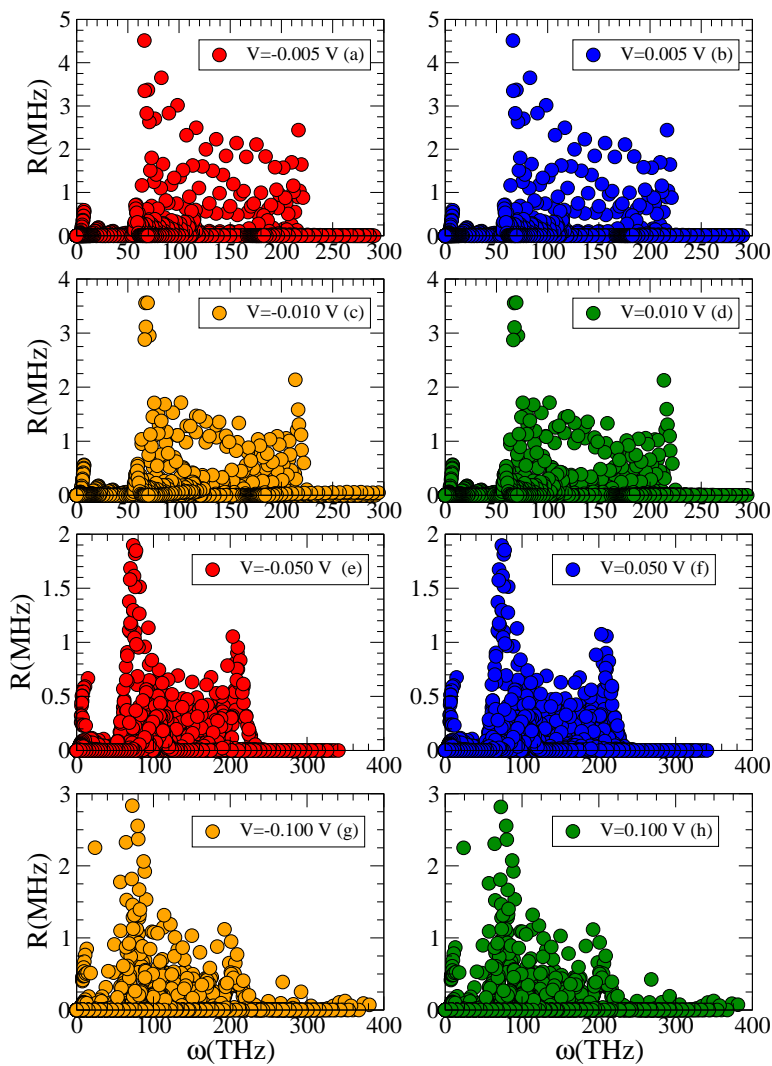

Figura 4: La distribuciones de probabilidades por unidad de tiempo para la emisión son mostradas para superredes de 20 pozos de GaAs de $150 \AA$ y 19 barreras de $\mathrm{Al}_{0.35} \mathrm{Ga}_{0.75} \mathrm{As}$ de $10 \AA$ de espesor, para diferentes diferentes de potenciales entre las barreras extremas. 


\section{Absorción y emisión de superredes dimeri- zadas}

Para las superredes dimerizadas de la forma ABAB... ABAB como se muestra en la figura 2 se calculan las probabilidades por unidad de tiempo de las transiciones de absorción entre la primera y la segunda minibanda, Fig.4(a); entre la segunda y tercera minibanda, Fig.4(b); entre la tercera y cuarta minibanda, Fig.4(c); entre la cuarta y la quinta minibanda, Fig.4(d); entre la quinta y sexta minibanda, Fig.4(e) y entre todas las minibandas, Fig.4(f).
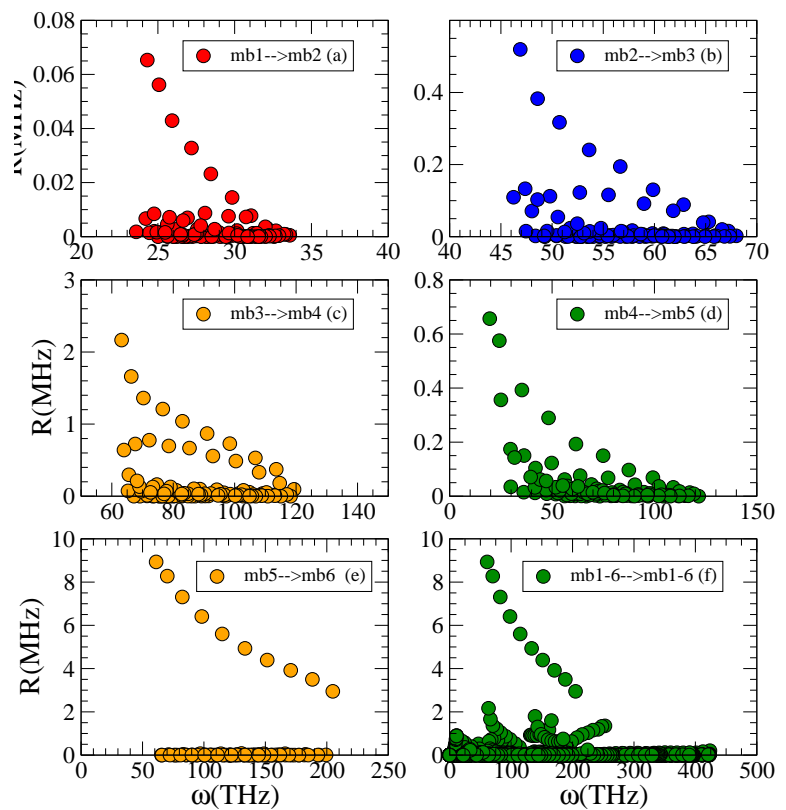

Figura 5: Las distribuciones de probabilidades por unidad de tiempo de la absorción entre las seis minibandas de una superred dimerizada con pozos cuánticos de $100 \AA$ y $150 \AA$ separados por barreras iguales de $10 \AA$ son mostradas. El detalle de las distribuciones se discute en el texto principal.

Se puede observar que las distribuciones de las probabilidades por unidad de tiempo entre la primera y la segunda minibanda, Fig.5(a), y entre la quinta y la sexta minibanda, Fig.5(e), tienen el mismo comportamiento de un decaimiento exponencial dados por $R=$ $46.89 \exp [-0.26905 \omega]$ y $R=13.585 \exp [-0.007403 \omega]$, en el rango de frecuencias de 23 a $34 \mathrm{THz}$ y de 60 a $200 \mathrm{THz}$, respectivamente. Ese mismo decaimiento exponencial se puede observar en las demás transiciones, Fig.5(b,c,d), pero también otras transiciones de media amplitud ocurren en los mismos rangos de frecuencia. Esto se explica por el hecho de que la tercera, cuarta y quinta minibandas de la superred dimerizada está conformada por autoestados pertenecientes a pozos de 100 A y $150 \AA$ que compiten en su acoplamiento para con- formar las minibandas teniendo una memoria del enmarañamiento de pertenecer a los pozos de $100 \AA$ y $150 \AA$. Por otro lado, se observa en la tabla 1, que la primera minibanda estrictamente pertenece a los pozos de 150 $\AA$, mientras que los autoestados de la sexta minibanda están intensamente acoplados porque energéticamente los autovalores de los pozos de $100 \AA$ (244.15-293.07 meV) y $150 \AA$ (239.07-293.14 meV) se superponen de modo que esa identidad monoenergética se manifiesta en las transiciones entre la quinta y la sexta minibanda, Fig.5(e) y que prevalece si lo comparamos a las otras distribuciones, Fig.5(f).
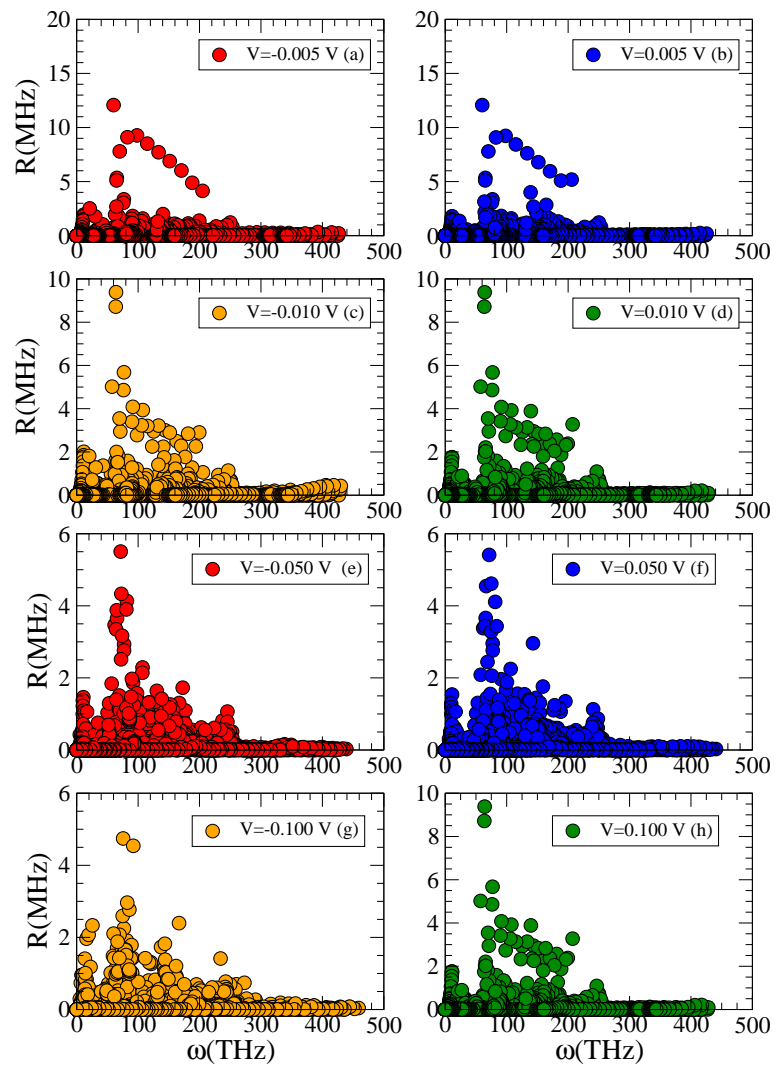

Figura 6: Las distribuciones de probabilidades por unidad de tiempo para las superredes dimerizadas con pozos de 100 y $150 \AA$ son mostradas para diferentes voltajes aplicados entre las barreras extremas de $\mathrm{Al}_{0.35} \mathrm{Ga}_{0.65} \mathrm{As}$.

$\mathrm{Al}$ aplicar una diferencia de potencial de $\pm 5 \mathrm{mV}$ entre las barreras extremas de $\mathrm{Al}_{0.35} \mathrm{Ga}_{0.65} \mathrm{As}$ se observa, en la Fig.6(a,b), que las distribuciones de las probabilidades por unidad de tiempo con decaimiento exponencial para $\mathrm{V}=0$, prácticamente se linealiza en el rango entre 80 y $200 \mathrm{THz}$ y aparece un pico aproximadamente a $60 \mathrm{THz}$ que va disminuyendo de altura conforme se aumenta el voltaje hasta $\pm 100 \mathrm{mV}$. También observamos que aparecen picos menores a 10 y $250 \mathrm{THz}$. La 
gran cantidad de puntos que se observa entre 60 y 250 $\mathrm{THz}$ con amplitudes de probabilidades por unidad de tiempo que alcanza los $2 \mathrm{MHz}$ se debe a las transiciones entre la segunda, tercera, cuarta y quinta minibanda, donde la memoria de la correlación entre los pozos 100 y $150 \AA$ juega un rol importante, Fig.6(c,d,e,f,g,h). Para las redes dimerizadas se observa que las distribuciones no son tan simétricas respecto al voltaje, positivo y negativo, aplicado. Esto se debe a que cuando el voltaje es negativo el primer pozo cerca al contacto de potencial $0 \mathrm{~V}$ es de $100 \AA$ y cuando es positivo el primer pozo es de $150 \AA$. Por tanto no existe simetría en cuanto a dimerización cuando un voltaje es aplicado. Si bien la dimerización genera mayores valores de las probabilidades por unidad de tiempo, sin embargo, luminesce con relativa intensidad en torno a $60 \mathrm{THz}$ para un rango de voltaje entre 0 y $100 \mathrm{mV}$.

\begin{tabular}{|c|c|c|c|c|c|c|c|c|}
\hline $\begin{array}{r}\text { PQ } 100 \AA \\
(\mathrm{meV})\end{array}$ & $\begin{array}{r}\text { PQ } 150 \AA \\
(\mathrm{meV})\end{array}$ & $\begin{array}{r}\text { Dímero } \\
(\mathrm{meV})\end{array}$ & $\begin{array}{l}\text { Minibandas } \\
\text { Dim. (meV) }\end{array}$ & $\begin{array}{r}\Delta_{d} \\
(\mathrm{meV})\end{array}$ & $\begin{array}{r}E_{\mathrm{mgd}} \\
(\mathrm{meV})\end{array}$ & $\begin{array}{r}\text { Minibandas } \\
(\mathrm{meV})\end{array}$ & $\begin{array}{r}\Delta \\
(\mathrm{meV})\end{array}$ & $\begin{array}{r}E_{\mathrm{mg}} \\
(\mathrm{meV})\end{array}$ \\
\hline \multirow{3}{*}{41.48} & 20.41 & 19.28 & $17.38-19.19$ & 1.81 & 17.38 & $15.22-22.83$ & 7.61 & 15.22 \\
\hline & & 38.81 & $34.71-39.49$ & 4.78 & 15.52 & & & \\
\hline & 80.12 & 77.77 & $69.92-79.47$ & 9.55 & 30.43 & $60.30-91.20$ & 30.90 & 37.47 \\
\hline \multirow[t]{2}{*}{155.45} & & 139.70 & $121.06-148.47$ & 27.41 & 41.59 & & & \\
\hline & 172.65 & 181.95 & $161.36-201.10$ & 39.74 & 12.89 & $134.51-204.61$ & 70.10 & 43.31 \\
\hline 285.15 & 274.80 & 263.64 & 241.29-296.19 & 54.90 & 40.19 & $239.07-\ldots \ldots$ & $\ldots \ldots$ & 34.46 \\
\hline
\end{tabular}

Tabla 1: Los autovalores de energía que corresponden a las minibandas formadas en la banda de conducción de las superredes de $\mathrm{GaAs} / \mathrm{Al}_{0.35} \mathrm{Ga}_{0.75} \mathrm{As}$. Se muestras los autovalores de energía calculados de pozos cuánticos (PQ) de $100 \AA$ y $150 \AA$ de un dímero conformado por dos PQs de 100 y $150 \AA$, respectivamente. $\Delta$ representa el ancho de una minibanda y $E_{\mathrm{mg}}$ representa el mini-gap de energía entre dos minibandas.

\section{Conclusiones}

El método usado en el presente trabajo permite simular el espectro de emisión de los láseres de cascada cuántica cuando las superredes tiene un sólo tipo de pozos cuánticos de GaAs de 150 Å. Estos luminescen a $6.582,46.074$ y $138.222 \mathrm{meV}$, correspondiendo a 10, 70 y $210 \mathrm{THz}$, en un rango de voltajes aplicados entre \pm 10 $\mathrm{y} \pm 100 \mathrm{mV}$.

Las barreras intermediarias escogidas son de $10 \AA$, aproximadamente 4 monocapas atómicas de $\mathrm{Al}_{0.35} \mathrm{Ga}_{0.65} \mathrm{As}$, lo que impide que al aplicar el voltaje exista una acumulación importante de cargas en los pozos que deformen via la ecuación de Poisson el fondo de la banda de conducción. El modelo funciona bien aún cuando las barreras son de dos monocapas atómicas y el número de pozos sea de cien. La distribuciones convergen a lorentzianas para 20, 40 y $250 \mathrm{THz}$.

Cuando se va incrementando el voltaje las minibandas más profundas de los pozos se superponen y se $m e$ talizan.
Las superredes dimerizadas presentan una distribución de probabilidades por unidad de tiempo asimétricas respecto a la diferencia de potencial aplicado positiva y negativamente. Y no ofrece una versatilidad de emisión para láseres de cascada cuántica cuando la dimerización es con pozos de 100 y $150 \AA$. Pero, sospechamos que en estas regiones de energía juega un papel importante el enmarañamiento cuántico debido a que en la segunda, tercera, cuarta y quinta minibanda de la superred dimerizada, los autoestados de los pozos de 100 y $150 \AA$, compiten para establecer la identidad propia de sus propios pozos, en las minibandas dimerizadas. Aquí se abre una posibilidad en el futuro para investigar esta inquietud.

\section{Agradecimientos}

Los autores agradecen al Vicerrectorado de Investigación y al Consejo Superior de Investigaciones de la UNMSM el soporte financiero mediante el proyecto $\mathrm{N}^{\mathrm{O}}$ 111301041.

\section{Referencias}

[1] L. Esaki y R. Tsu; Superlattice and negative differential conductivity in semiconductors, IBM J. Res. Devel. 14, 61 (1970).
[2] R. N. Hall, G. E. Fenner, J. D. Kingsley, T. J. Soltys y R. O. Carlson; Coherent light emission from GaAs junctions, Phys. Rev. Lett. 9, 366 (1962).

[3] Roberto Paiella, Intersubband transitions in quan- 
tum structures, McGraw-Hill, New York (2006).

[4] J. Faist, F. Capasso, D. L. Sivco, C. Sirtori, A. L. Hutchinson y A. Y. Cho; Quantum cascade laser, Science 264, 553 (1994).

[5] F. T. Vasko y V. V. Mitin, Electronic states in heterostructures formed by ultranarrow layers, arxiv:cond-mat, $1110.0744 \mathrm{v} 1$ (2011).

[6] Peter Y. Yu y Manuel Cardona, Fundamentals of Semiconductors: Physics and Materials Properties, cap. 9, cuarta edición, Springer-Verlag, Berlín (2010).

[7] Chihiro Hamaguchi, Basic Semiconductors Phy- sics, cap 8, segunda edición, Springer-Verlag, Berlín (2010).

[8] Supriyo Data, Electronic Transport in Mesoscopic Systems, Cambridge University Press, Londres (1995).

[9] J. S. Townsend, A Modern Approach to Quantum Mechanics, segunda edición, University Science Books, Sausalito (2000).

[10] Paul Strange, Relativistic Quantum Mechanics with applications to condensed matter and atomic physics, Cambridge University Press \& Beijing World Publishing Corporation, Beijing (2008). 